\title{
El tratamiento con gliburida en mujeres con Diabetes Gestacional es una alternativa clínicamente efectiva
}

A comparison of Glyburide and Insulin in women with gestational Diabetes Mellitus. Langer O, Conway DL, Berkus MD. N Eng/ J Med 2000;343:1134-8

\section{Objetivo}

Comparar el tratamiento habitual con insulina con el realizado con gliburida en embarazadas con respuesta insatisfactoria a la dieta y el ejercicio.

\section{Diseño}

Ensayo clínico controlado doble ciego.

Lugar

Maternidades de San Antonio, Texas.

\section{Pacientes}

Embarazadas $(n=404)$ con diagnóstico de Diabetes Gestacional por sobrecarga con 100grs. de glucosa con dos valores alterados, edad entre $18-40$ años; $83 \%$ hispanas, $12 \%$ blancas no hispanas, $5 \%$ negras; todas pertenecientes a Medicaid y de buen nivel aducativo.

\section{Intervención}

Las pacientes fueron randomizadas a tratamiento convencional con insulina ( $n=203$ ) comenzando con $0.7 \mathrm{U} / \mathrm{kg} /$ día o a tratamien- to con gliburida ( $n=201$ ) con dosis inicial de $2.5 \mathrm{mg}$. en aumento hasta $20 \mathrm{mg}$. hasta alcanzar glucemia en ayunas de $95 \mathrm{mg} / \mathrm{dl}$ y postpandial de $120 \mathrm{mg} / \mathrm{dl}$ o menores.

\section{Medición de resultados principales}

El resultado principal fue adecuado control glucémico (glucemia media: 90-105 mg/dl; prepandial <95mg/dl; postpandial <120 mg/dl)

\section{Resultados principales}

La media de glucemia en el grupo gliburida fue de $105 \pm 16 \mathrm{mg} / \mathrm{dl}$ en tanto en el grupo insulina fue de $105 \pm 18 \mathrm{mg} / \mathrm{dl}(\mathrm{P}=0.99)$. El 4 $\%$ de las pacientes en el grupo gliburida requirió insulina para su adecuiado control metabólico.

También se evaluó: peso para edad gestacional, macrosomía hipoglucemia neonatal, membrana hialina (criterios de Corbet), insulinemia de cordón, ictericia neonatal, policitemia e hipocalcemia, sin encontrar diferencias significativas entre ambos grupos.

\section{Conclusión}

En mujeres con diabetes gestacional la gliburida es una opción terapeútica segura y clínicamente efectiva.

\section{Comentario}

La Diabetes Gestacional continúa siendo tema de debate ya que no existe acuerdo sobre el beneficio inequívoco de un tratamiento costoso y no siempre efectivo ${ }^{1}$. Existe evidencia sufieciente que el primer escalón de tratamiento es la dieta y el ejercicio. Si no se alcanza el control glucémico adecuado debe agregarse insulina 2

Existe recomendación de no usar hipoglucemiantes orales por el potencial desarrollo de malformaciones, hipoglucemia neonatal, policitemia e hipocalcemia ${ }^{1}$ Con respecto a la primera afirmación la diabetes gestacional se diagnostica habitualmente una vez finalizada la organogénesis, por lo que es poco probable que se pueda atribuir la mayor incidencia de malformacioes a estas drogas,

teniendo sí relación con la hiperglucemia en las primeras semanas de gestación. Las siguientes complicaciones neonatales son todas derivadas del hiperinsulinismo fetal. Elliot y colaboradores demostraron en estudios de laboratorio que la gliburida no atraviesa la placenta, por lo que no puede considerarse causa de estas alteraciones neonatales ${ }^{3}$. Hasta ahora el uso de hipoglucemiantes orales durante el embarazo aparece como contraindicación. Basándonos en la evidencia existente y en los resultados de este estudio se puede considerar a la gliburida como un opción terapeutica en el manejo de la Diabetes Gestacional 4

Queda por definir si este efecto se puede hacer extensivo a otras sulfonilureas utilizadas comunmente en nuestro medio.

\section{Dra. María Natalia Basualdo [ Hospital Materno Infantil R. Sardá. Ciudad de Buenos Aires}

\section{Referencias}

1- MetzgerBE, Coustan DR.Summary and Recommendations of the fourth International Workshop-Conference on Gestational Diabetes. Diabetes Care 1998;21:supl 2: 161-167. 2- Diabetes and Pregnancy. ACOG technical bulletin № 200. Washington D.C: American College of Obstetricians and Gynecologists, December 1994: 359-66.

3- Elliot BD, Schenker S, Langer O. Comparative placental transport of oral hypoglycemic agents in humans: a model of human placental transfer. Am J Obstet Gynecol 1994;171:653-60 4- Greene MF: Oral hypoglycemic drugs for Gestational Diabetes. N Engl J Med. 2000, 16: 1178.

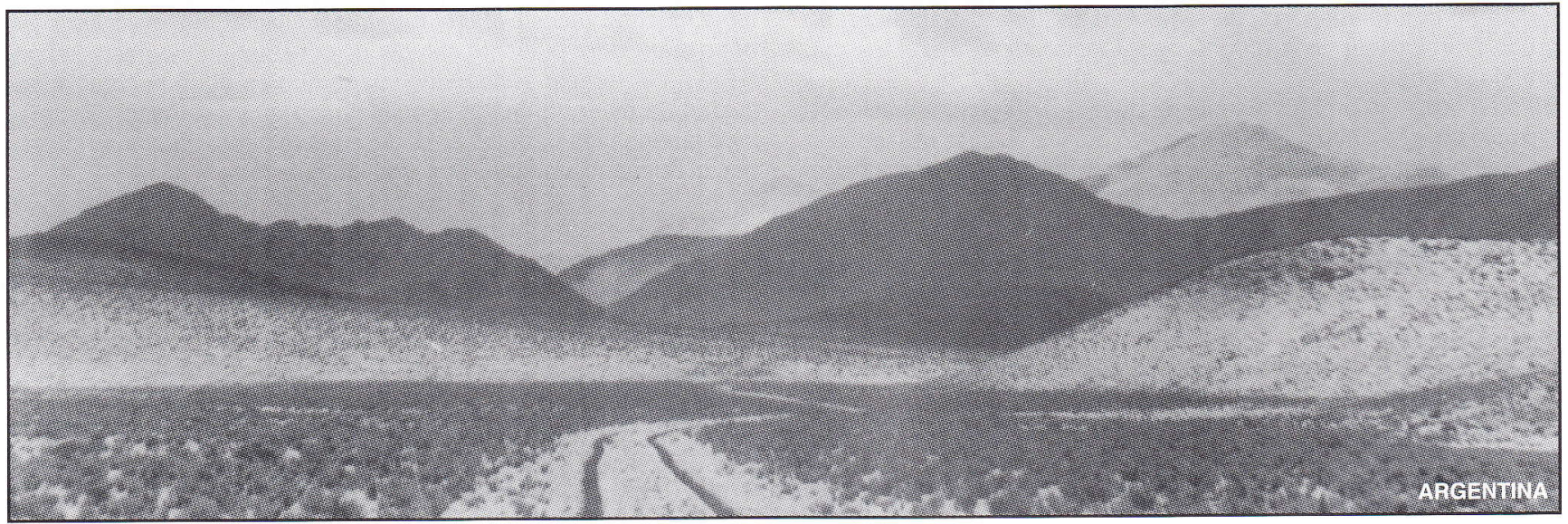

